\title{
Electrocardiogram Original Result
}

National Cancer Institute

\section{Source}

National Cancer Institute. Electrocardiogram Original Result. NCI Thesaurus. Code C83104.

The outcome of the electrocardiogram assessment. 